\title{
Magnetic Resonance Imaging as a Determinant for Surgical Release of Congenital Muscular Torticollis: Correlation with the Histopathologic Findings
}

Jee Hyun Hwang, M.D., Han Byul Lee, M.D., Jang-Hee Kim, M.D., M.S. ${ }^{1}$, Myong Chul Park, M.D., Ph.D. ${ }^{2}$, Kyu-Sung Kwack, M.D., Ph.D. ${ }^{3}$, Jae Deok Han, M.D., Shin-Young Yim, M.D., Ph.D.

The Center for Torticollis, Departments of Physical Medicine and Rehabilitation, ${ }^{1}$ Pathology , ${ }^{2}$ Plastic and Reconstructive Surgery, ${ }^{3}$ Radiology, Ajou University School of Medicine, Suwon 443-721, Korea

Objective (1) To present the magnetic resonance imaging (MRI) findings of congenital muscular torticollis (CMT) of subjects who underwent surgical release and subjects who showed a good prognosis with stretching exercises and (2) to correlate the MRI findings with the histopathologic findings of CMT for subjects who underwent surgical release in order to examine the hypothesis that the MRI findings of CMT can be used as a determinant to perform surgical release of CMT.

Method The neck MRI findings of 33 subjects who underwent surgical release for CMT were compared with those of 18 subjects who were successfully managed only with conservative management. The MRI findings were correlated with the histopathologic sections of the CMT mass.

Results All 33 subjects (100\%) who underwent surgical release showed one or more low signal intensities within the involved sternocleidomastoid muscle (SCM) on the T1- and T2-weighted images of neck MRI. The eighteen non-surgical candidates showed only enlargement of the SCM without low signal intensity within the SCM. The histopathologic findings showed interstitial fibrosis and/or the presence of aberrant tendon-like excessive dense connective tissue that was either well-arranged or disorganized.

Conclusion The histopathologic findings and MRI findings showed good correlation in terms of the amount of fibrosis and aberrant dense connective tissue within the SCM. If multiple or large low signal intensities within the SCM are noted, we think that surgical release should be considered.

Key Words Congenital torticollis, Magnetic resonance imaging (MRI), Fibrosis

Received March 6, 2012; Accepted April 25, 2012

Corresponding author: Shin-Young Yim

The Center for Torticollis, Department of Physical Medicine and Rehabilitation, Ajou University School of Medicine, 206 WorldCup-ro, Yeongtong-gu, Suwon 443-721, Korea

Tel: +82-31-219-5284, Fax: +82-31-219-5209, E-mail: syyim@ajou.ac.kr (a) This is an open-access article distributed under the terms of the Creative Commons Attribution Non-Commercial License (http:// creativecommons.org/licenses/by-nc/3.0) which permits unrestricted noncommercial use, distribution, and reproduction in any medium, provided the original work is properly cited.

Copyright $\odot 2012$ by Korean Academy of Rehabilitation Medicine

\section{INTRODUCTION}

Congenital muscular torticollis (CMT) is one of the most common musculoskeletal problems in children and the prevalence is as high as $3.92 \% .^{1,2}$ In patients with CMT, shortening of the unilateral sternocleidomastoid muscle (SCM) causes ipsilateral head tilt, slight rotation of the chin to the contralateral side and limitations in head movement, ${ }^{2}$ and if left untreated, it can cause 
musculoskeletal complications such as craniofacial deformities and compensatory scoliosis. ${ }^{3}$ The mainstay of management of CMT is stretching exercise. About $90 \%$ or more of CMT cases are known to be cured with only stretching exercise and without any musculoskeletal complications. ${ }^{4}$ However, about $10 \%$ of the cases of CMT do not respond to stretching exercise and the CMT causes secondary musculoskeletal deformities that have a negative impact on both the psychological and social aspects of life. However, not much is known about what determines the responsiveness to stretching exercise for treating CMT.

The use of magnetic resonance imaging (MRI) for the musculoskeletal system has increased. While there are some reports on the MRI findings of $\mathrm{CMT}^{5-12}$ the role of MRI in CMT has been controversial. ${ }^{9}$ Furthermore, only a few studies have investigated the correlation between the neck MRI findings and the histopathologic findings of a CMT mass in the SCM. ${ }^{11,12}$ We have experienced a couple of patients with CMT who did not respond to stretching exercise and showed craniofacial asymmetry. The neck MRI showed that low signal intensities were found within the shortened SCM. These clinical experiences prompted us to hypothesize that the MRI findings of CMT could be used as a determinant for performing surgical release of CMT. We obtained more neck MRIs of CMT especially from subjects who needed surgical release, and we correlated the MRI findings with their histopathologic findings.

The purposes of this study were (1) to present the MRI findings of CMT of subjects who underwent surgical release as well as of subjects who showed a good response to stretching exercises and (2) to correlate the MRI findings with the histopathologic findings of CMT for subjects who underwent surgical release in order to examine the hypothesis that the MRI findings of CMT can be used as a determinant for performing surgical release of the CMT.

\section{MATERIALS AND METHODS}

This was a retrospective study and we used information from the medical records in this study. The data included the demographics and the radiologic and histopathologic studies of the study population. This study was approved by the Institutional Review Board (IRB) of our medical center. Informed consent from patients was not necessary because this was a retrospective study using only the medical records.

\section{Enrollment of patients}

Among the subjects who came to our medical center for abnormal posture of the head and neck from January 2009 to October 2009, the subjects who met all of the following criteria were included in this study: (1) the subjects were diagnosed with CMT according to the diagnostic criterion of CMT mentioned below, (2) neck MRI without contrast for CMT was taken when the subjects were 4 months old or older, and (3) there was a followup period of 6 months or more since taking neck MRI. Therefore, all the subjects were 10 months old or older at the time of this study. We made an algorithm to show the enrollment of subjects in this study (Fig. 1).

\section{Diagnosis of CMT}

In this study, CMT was diagnosed for subjects who showed (1) shortening of the unilateral SCM, with limitation of the full range of motion of lateral flexion toward the ipsilateral shoulder and slight rotation of the chin to the contralateral side; and (2) lack of full rotation of the neck toward the contralateral shoulder, which was noticed since childhood (3) without any postnatal history of injury or surgery of the neck. Among the subjects who were diagnosed by the above definition, although the majority of subjects showed a thickened unilateral SCM that looked like a tumor or mass, the older subjects showed a shortened unilateral SCM with the appearance of a taut cord-like band without a mass on the unilateral SCM. For this reason, the presence of a thickened SCM or the presence of a mass on the unilateral SCM was not included in the diagnostic criterion of CMT.

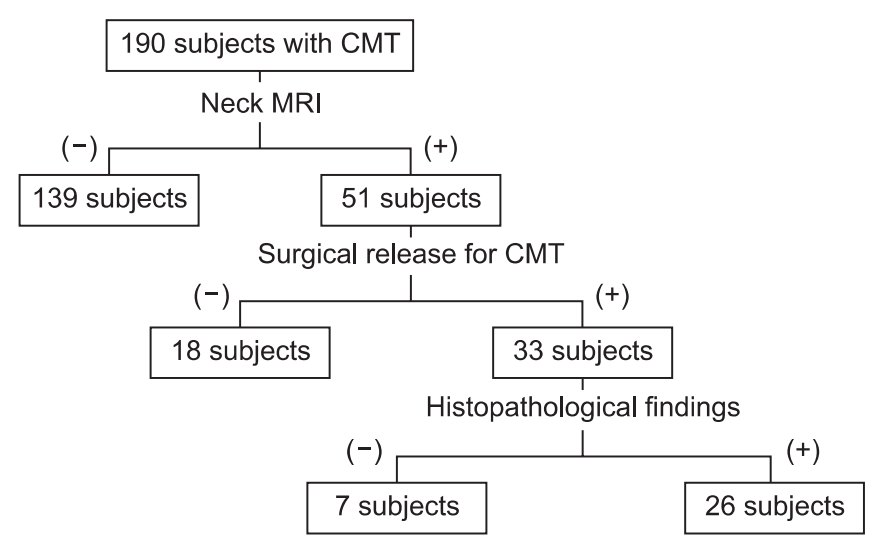

Fig. 1. The algorithm for enrollment of subjects from January 2009 to October 2009. 


\section{Magnetic resonance imaging}

Most of the neck MRIs were performed in order to determine whether or not surgical release was required. The majority of the subjects who showed full range of motion (ROM) of the neck with/without a mass at the age of 4 months old did not undergo neck MRI. Our previous clinical experience as well as the reports of previous studies $^{7,8}$ showed that the neck MRIs of subjects younger than 4 months old demonstrate high signal intensity on $\mathrm{T} 2$ and proton density-weighted images (Fig. 2). This high signal intensity disappears as the infants get older, and at the age of 4 months, those high signal intensities can no longer be observed. This was the rationale for taking neck MRI at the age of 4 months or older in this study. We checked the neck MRI at the age of 4 months or older if there were limitations of neck flexion and rotation despite intensive stretching exercises for 3-6 months. The exception to this was longstanding neglected CMT subjects in whom neck MRIs were performed without a follow up period of physical therapy. We thought that an early diagnosis of CMT and a decision about surgical release of SCM was important to prevent the progress of secondary musculoskeletal complications in these neglected subjects. The neck MRIs were obtained using one of two 1.5-T MRI units (Signa, GE Healthcare). The following imaging parameters were used: TR/TE, 400600/11-14 for T1-weighted imaging and 3,000-4,000/75100 for T2-weighted imaging; section thickness, 3.0-6.0 mm; slice gap, 1-2.5 mm; and FOV, 180-240 mm depending on body size and section planes. The neck MRIs of the subjects were reviewed by both a doctor with over 10 years of experience with CMT patients and a radiologist specializing in the musculoskeletal system. The major review points were as follows:

1) The presence of low signal intensity within the shortened SCM (compared with the signal intensity in the contralateral SCM)

2) Identification of the head(s) involved: on neck MRI, both the sternal and clavicular heads were well visualized. The involved head(s) such as the sternal head, the clavicular head or both heads of the SCM were identified in terms of the signal intensity within the shortened SCM

3) Measurement of the thickness of the shortened SCM: if the shortened SCM was $2 \mathrm{~mm}$ or more thicker than the SCM of the contralateral side, it was considered thickened. If the shortened SCM was $2 \mathrm{~mm}$ or more thinner than the SCM of the contralateral side, it was considered atrophied. If the difference between the right SCM and the left SCM was $2 \mathrm{~mm}$ or less, then the difference was considered negligible.

\section{Surgical release for CMT}

Surgical release for CMT was performed on patients (1) who were aged 6 months or older and showed significant functional limitation of the neck motion due to the shortening of the unilateral SCM despite stretching exercises for 6 months or (2) who were longstanding neglected CMT cases already showing obvious craniofacial asymmetry. Under general anesthesia, abnormal fibrous tissue and tight fascia was released and the involved head(s) such as the sternal head, the clavicular head or both heads was/were released by the third author.

\section{Review of the histopathologic findings}

After surgical release of the shortened SCM, the histopathologic sections of the CMT in the SCM were reviewed. All muscle specimens for histopathologic study were obtained from the lower end of the sternocleidomastoid muscle because the myotomy or myectomy was done through a skin incision just above the upper margin of the clavicle.
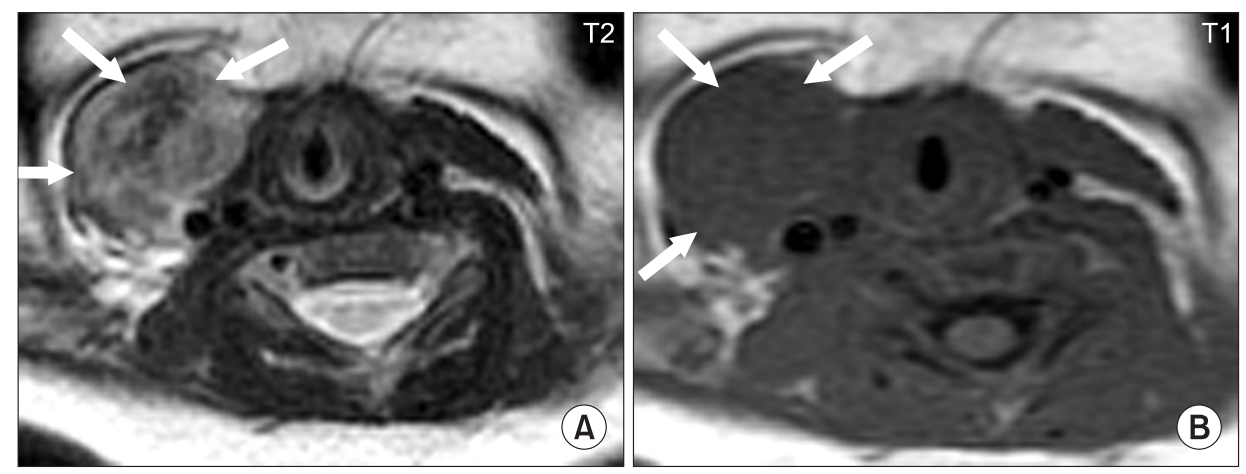

Fig. 2. The neck MRI findings of a one month old girl with right congenital muscular torticollis show high signal intensity on the (A) T2weighted image compared to the (B) T1-weighted image (arrows). 


\section{RESULTS}

Fifty one subjects underwent neck MRI for CMT from January 2009 to October 2009 (28 men and 23 women; age at the time of neck MRI: $59.63 \pm 103.55$ months old; age range: 4 months-38 years). Thirty three among the 51 subjects underwent surgical release.

The presence of low signal intensity within the shortened SCM

All 33 subjects who underwent surgical release (100\%) showed one or more low signal intensities within the in- volved SCM on both the T1- and T2-weighted images of neck MRI. Compared to the MRI findings of subjects that did not have low signal intensities within the SCM (Fig. 3), the images of these subjects showed the typical findings of low signal intensities within the SCM (Fig. 4). Eighteen subjects with CMT were successfully managed with physical therapy including stretching exercise, ending up with restoration of the full range of motion of the neck and decrease of the size of the mass.

\section{Identification of head(s) involved}

Low signal intensities were found in the sternal head of
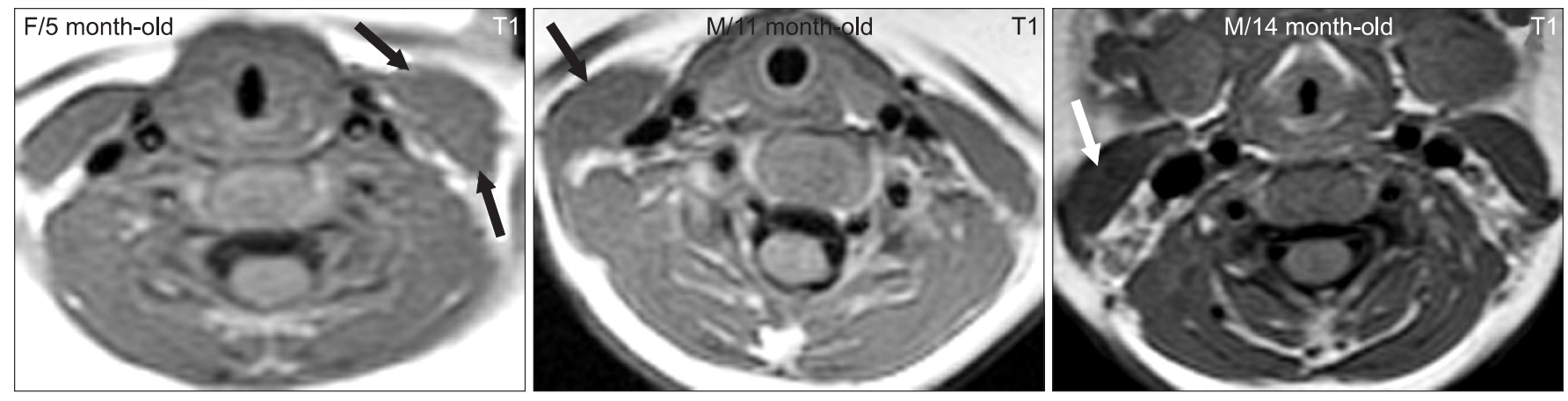

Fig. 3. Typical neck MRI findings of subjects who show a good response to stretching exercises. Thickened sternocleidomastoid muscles do not have low signal intensities (arrows).
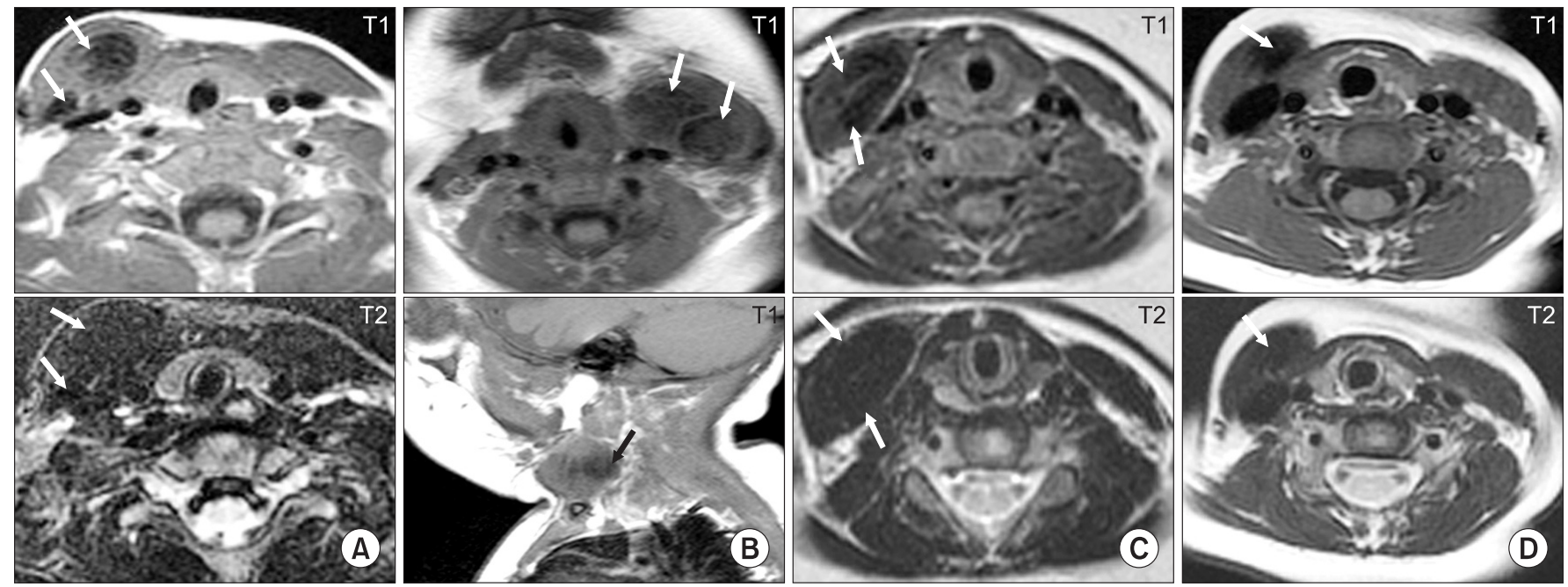

Fig. 4. Typical neck MRI findings of subjects who underwent surgical release for congenital muscular torticollis (CMT). (A) Eleven month old boy with right CMT showing low signal intensities on the T1- and T2-weighted axial images of both the sternal head and clavicular head (arrows) of the right sternocleidomastoid muscle (SCM). (B) Four month old girl with left CMT showing low signal intensities on the T1-weighted axial and sagittal images of the left SCM (arrows). (C) Six month old girl with right CMT showing low signal intensities on both the T1- and T2-weighted axial images of the right SCM (arrows). (D) Eighteen month old girl with right CMT showing low signal intensity on both the T1- and T2-weighted axial images of the right SCM (arrows). 
the SCM in 21 subjects (63.6\%), in the clavicular head in $3(9.1 \%)$, and in both the sternal and clavicular heads in 9 (27.3\%) among the 33 subjects with low signal intensities within the SCM. Therefore, the sternal head was involved in $90.9 \%$ of the total subjects with CMT.
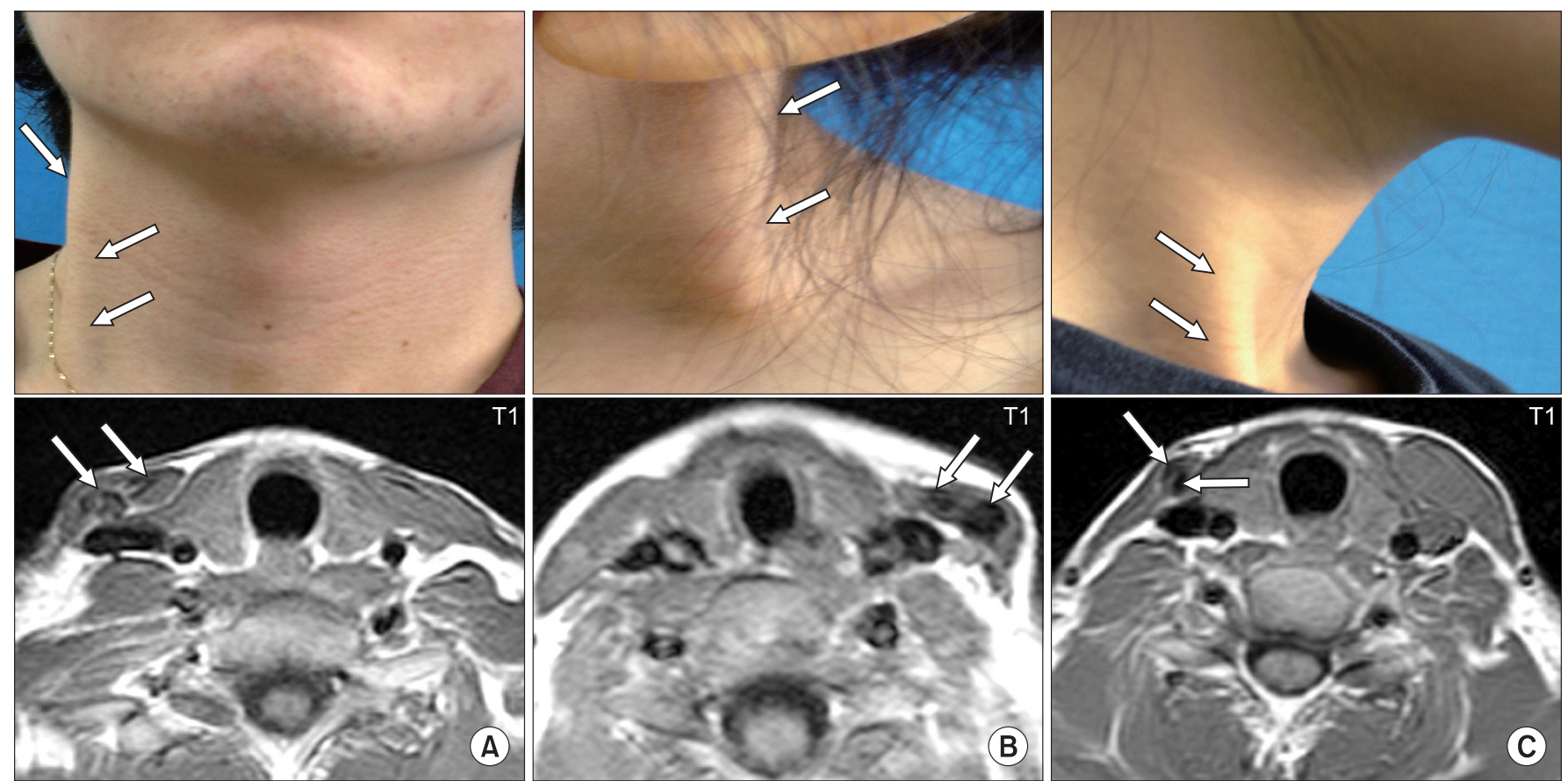

Fig. 5. Pictures showing subjects with atrophy or same thickness of the shortened sternocleidomastoid muscle (SCM) with low signal intensities on the T1-weighted axial images of the SCM. (A) Twenty-two year old man with right congenital muscular torticollis (CMT) showing a cord-like right SCM (arrows). Neck MRI showed low signal intensities (arrows) and did not show significant difference of thickness between the right and left SCM. (B) Five year old girl with left CMT showing a cord-like left SCM (arrows) and low signal intensity (arrows) on left SCM. (C) Twenty year old woman with right CMT showing atrophied cord-like right SCM (arrows) with low signal intensity (arrows) on right SCM.
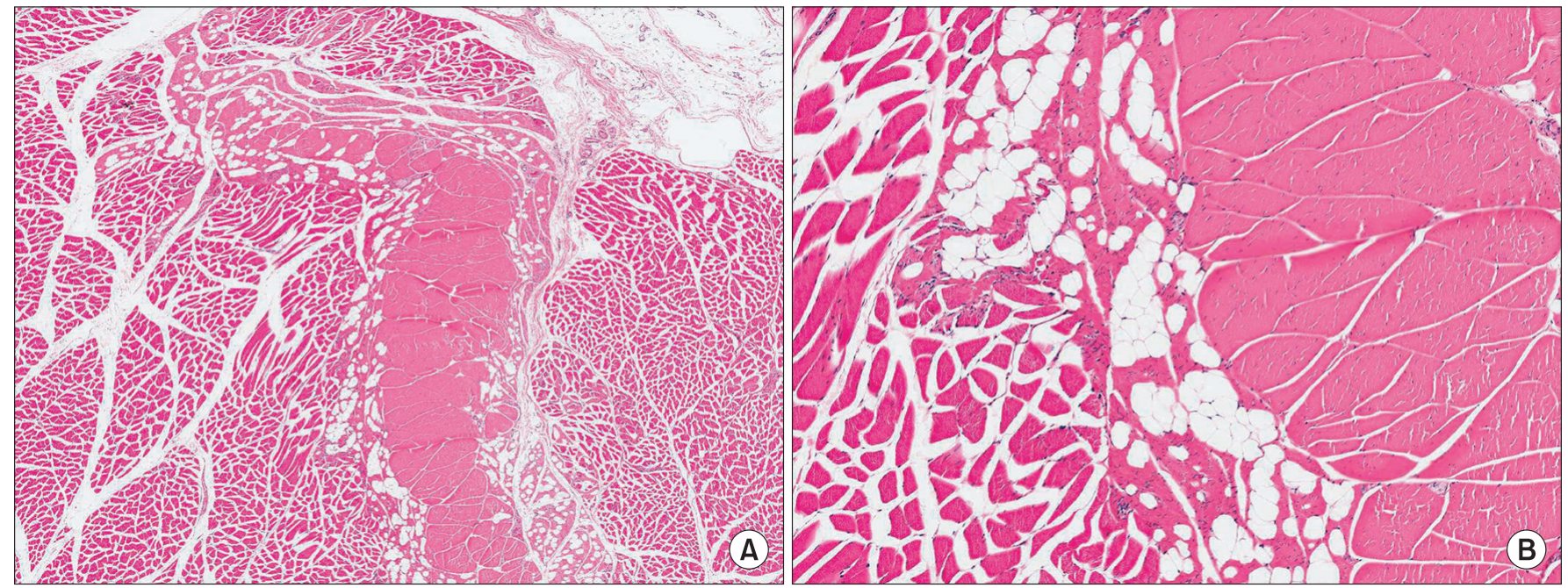

Fig. 6. The histologic findings of the normal sternocleidomastoid muscle without congenital muscular torticollis (H\&E, (A) $\times 100$; (B) $\times 200)$. 


\section{Measurement of the thickness of the shortened SCM}

In terms of the thickness of the shortened SCM, 6 subjects whose ages were between 10 months to 38 years old did not show a thickened SCM among 51 subjects who underwent neck MRI for CMT and showed atrophy or the same thickness of the shortened SCM with low signal intensity in the shortened SCM (Fig. 5). These subjects presented clinically with a cord-like prominent unilateral SCM with limitation of neck motion and craniofacial asymmetry.

\section{Review of the histopathologic findings}

Among the 33 subjects who underwent surgical release, the histopathologic sections of the CMT from the surgical release of SCM were available for 26 subjects ( 22 men and 13 women; age at the time of surgical release: $62.51 \pm 85.51$ months old; and age range: 6 months old-38 years). The normal SCM without CMT shows normal histologic findings (Fig. 6). Regarding the histopathologic findings of CMT (Fig. 7), all 26 subjects showed interstitial fibrosis and/or the presence of aberrant tendon-like excessive dense connective tissue that was either well-arranged or disorganized. Abnormally coiled vessels and myxoid change of the nerves were also noted in some subjects. There were 8 subjects $(30.8 \%)$ who showed prominent fat infiltration.
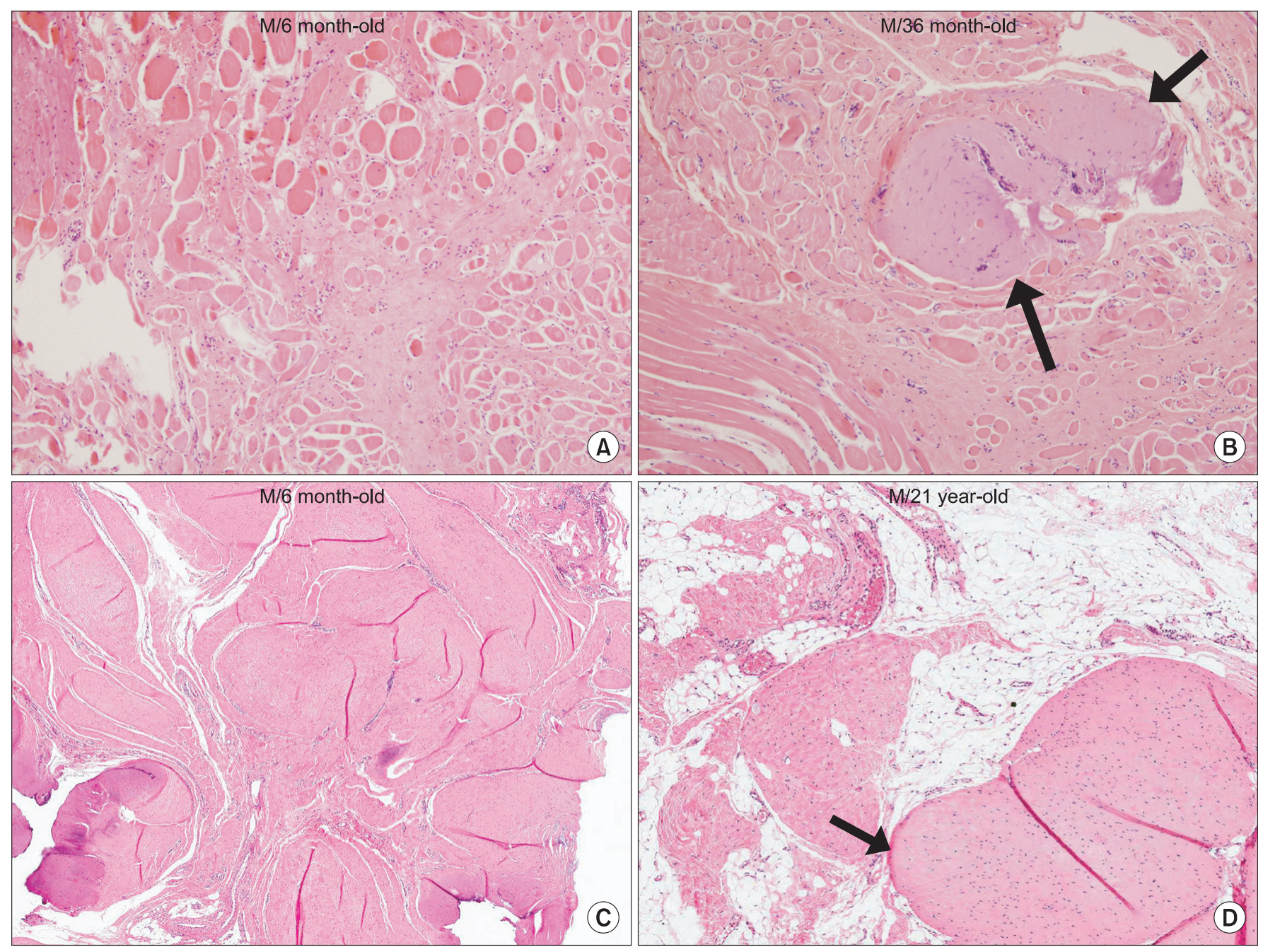

Fig. 7. The histopathological findings of the sternocleidomastoid muscle with congenital muscular torticollis. (A) Diffuse interstitial fibrosis with accompanying atrophic muscle fibers is noted (H\&E, $\times 200)$. (B) Interstitial fibrosis with presence of aberrant tendon-like excessive dense connective tissue (arrows) (H\&E, $\times 200)$. (C) Interstitial fibrosis with presence of aberrant tendon-like excessive dense connective tissue which was well-arranged (H\&E, $\times 40)$. (D) Aberrant tendon-like excessive dense connective tissue and prominent fat infiltration (arrow) (H\&E, ×40). 


\section{DISCUSSION}

Our current study suggested that neck MRI is useful in the process of determining whether surgical release is needed for CMT cases in which there is significant limitation of neck movement even with intensive stretching exercises for 3-6 months. What we are able to add to the previous findings is that if there are multiple or a large amount of low signal intensities within the SCM, surgical release for CMT should be considered. ${ }^{6-9,11,12}$ The histopathologic findings revealed that some of these low signal intensities were due to aberrant tendon-like excessive dense connective tissue that was either well-arranged or disorganized, along with interstitial fibrosis, a finding which has already been reported in the previous studies. ${ }^{11,12}$ Fibrosis leads to a reduction of the mobile proton (hydrogen ion) density, and so it shows hypointensity on both the T1- and T2-weighted MR images.

Not much is known about what determines the responsiveness of CMT to stretching exercise. If we know what the poor prognostic factors for the conservative management of CMT are, surgery can be considered for the cases with poor prognosis before the CMT causes secondary musculoskeletal deformity and the patient is overwhelmed by the stress from the stretching exercise for a prolonged period. Although an ultrasonogram has been used as the golden standard for the diagnosis of $\mathrm{CMT}^{1,4}$ its role in providing information on the prognosis of CMT seems limited. There have been several reports on the histopathologic findings of CMT, and most of them were mainly based on fine needle aspiration cytology of $\mathrm{CMT}^{10,13-20}$ and they did not give useful information in terms of the prognosis of CMT. It has been known that early detection and initiation of conservative treatment for CMT leads to resolution of CMT in the majority of patients. ${ }^{4,21,22}$ However, it is possible that some subjects who already have aberrant tendon-like excessive dense connective tissue within the SCM since birth will not show a favorable response even with early initiation of conservative management. This aberrant tendon-like excessive dense connective tissue within the SCM may function as an obstacle to proper muscle functioning of the surrounding SCM, and may eventually cause significant atrophy of the surrounding SCM as the subjects get older. The atrophied SCM looks like a cord or tendon because of the scarcity of proper muscle and the lack of contractility of this aberrant dense connective tissue.

The majority of the previous reports on the MRI findings of CMT were case reports or reports on a small number of patients. Parikh et al. ${ }^{9}$ enrolled 40 subjects with CMT in their study and concluded that MRI did not alter the treatment of these subjects. It is surmised that the different conclusions between our current study and Parikh's report is related to the number of surgical candidates who were included in the studies. Our study reported the MRI findings for 33 surgical candidates who showed a poor response to conservative treatment for CMT and they all showed large or multiple low signal intensities within the SCM. But $90 \%$ or more of the cases of CMT are known to respond well to conservative treatment and the majority of them would not have shown low signal intensity on neck MRI.

This study has several significant limitations. First, this was not a randomized controlled trial but a retrospective review. During the study period, most of the neck MRIs were taken in order to determine whether surgical release was required. Further study with more children who show a good response to conservative management is required. Second, the histopathologic findings of the SCM were only from the children who showed incomplete recovery with stretching exercise, which means that we do not know the pathological changes of the majority of the children who showed a good response to conservative management. Further, all the muscle specimens for histopathologic study were taken from the lower end of the SCM because we conducted myotomy or myectomy through a skin incision just above the upper margin of the clavicle. If the CMT was located far from the lower end of the SCM, the muscle block that had the characteristic findings was not submitted for histopathologic study. Third, most of the subjects in this study had been given conservative management before they came to our hospital. But some neglected CMT cases who showed craniofacial asymmetry and had not had any previous physical therapy were enrolled in our study, and all of them also showed low signal intensity and interstitial fibrosis and/or aberrant tendon like dense connective tissue in the SCM and underwent surgical treatment.

\section{CONCLUSION}

Even with these limitations, the histopathologic find- 
ings and MRI findings demonstrated good correlation with each other and we were able to conclude that low signal intensities represent interstitial fibrosis with/without aberrant dense connective tissue. Based on these findings, we suggest that low signal intensities within the SCM on MRI are a strong indication for surgical release.

\section{REFERENCES}

1. Chen MM, Chang HC, Hsieh CF, Yen MF, Chen TH. Predictive model for congenital muscular torticollis: analysis of 1021 infants with sonography. Arch Phys Med Rehabil 2005; 86: 2199-2203

2. Morrison DL, MacEwen GD. Congenital muscular torticollis: observations regarding clinical findings, associated conditions, and results of treatment. J Pediatr Orthop 1982; 2: 500-505

3. Yim SY, Lee IY, Park MC, Kim JH. Differential diagnosis and management of abnormal posture of the head and neck. J Korean Med Assoc 2009; 52: 705-718

4. Cheng JC, Wong MW, Tang SP, Chen TM, Shum SL, Wong EM. Clinical determinants of the outcome of manual stretching in the treatment of congenital muscular torticollis in infants. A prospective study of eight hundred and twenty-one cases. J Bone Joint Surg Am 2001; 83-A: 679-687

5. Ablin DS, Jain K, Howell L, West DC. Ultrasound and MR imaging of fibromatosis colli (sternomastoid tumor of infancy). Pediatr Radiol 1998; 28: 230-233

6. Brans J, Aramideh M, Bosch A, Speelman H. Late presentation of congenital muscular torticollis: a nondystonic cause of torticollis. J Neurol 1996; 243: 354356

7. Entel RJ, Carolan FJ. Congenital muscular torticollis: magnetic resonance imaging and ultrasound diagnosis. J Neuroimaging 1997; 7: 128-130

8. Hayashi S, Ito K, Kogure T, Shimada M, Tsubuku M, Kaneko I, Kusama K. Clinical evaluation of congenital muscular torticollis by using MR imaging. Nihon Igaku Hoshasen Gakkai Zasshi 1995; 55: 957-960

9. Parikh SN, Crawford AH, Choudhury S. Magnetic resonance imaging in the evaluation of infantile torticollis. Orthopedics 2004; 27: 509-515
10. Pereira S, Tani E, Skoog L. Diagnosis of fibromatosis colli by fine needle aspiration (FNA) cytology. Cytopathology 1999; 10: 25-29

11. Singer C, Green BA, Bruce JH, Bowen BC, Weiner WJ. Late presentation of congenital muscular torticollis: use of MR imaging and CT scan in diagnosis. Mov Disord 1994; 9: 100-103

12. Whyte AM, Lufkin RB, Bredenkamp J, Hoover L. Sternocleidomastoid fibrosis in congenital muscular torticollis: MR appearance. J Comput Assist Tomogr 1989; 13: $163-164$

13. Apple SK, Nieberg RK, Hirschowitz SL. Fine needle aspiration diagnosis of fibromatosis colli. A report of three cases. Acta Cytol 1997; 41: 1373-1376

14. Kurtycz DF, Logrono R, Hoerl HD, Heatley DG. Diagnosis of fibromatosis colli by fine-needle aspiration. Diagn Cytopathol 2000; 23: 338-342

15. Nayak SP, Munshi MM, Bobhate SK. Cytodiagnosis of fibromatosis colli. Cytopathology 2007; 18: 266-268

16. Park IS, Kim L, Choi SJ, Han JY, Kim JM, Chu YC, Choi SG. Fine needle aspiration cytologic findings of fibromatosis colli: a report of three cases. Korean J Cytopathol 2005; 16: 61-65

17. Sauer T, Selmer L, Freng A. Cytologic features of fibromatosis colli of infancy. Acta Cytol 1997; 41: 633-635

18. Schwartz RA, Powers CN, Wakely PE Jr, Kellman RM. Fibromatosis colli. The utility of fine-needle aspiration in diagnosis. Arch Otolaryngol Head Neck Surg 1997; 123: 301-304

19. Cheng JC, Metreweli C, Chen TM, Tang S. Correlation of ultrasonographic imaging of congenital muscular torticollis with clinical assessment in infants. Ultrasound Med Biol 2000; 26: 1237-1241

20. Gonzales J, Ljung BM, Guerry T, Schoenrock LD. Congenital torticollis: evaluation by fine-needle aspiration biopsy. Laryngoscope 1989; 99: 651-654

21. Jaber MR, Goldsmith AJ. Sternocleidomastoid tumor of infancy: two cases of an interesting entity. Int J Pediatr Otorhinolaryngol 1999; 47: 269-274

22. Cheng JC, Tang SP, Chen TM, Wong MW, Wong EM. The clinical presentation and outcome of treatment of congenital muscular torticollis in infants--a study of 1,086 cases. J Pediatr Surg 2000; 35: 1091-1096 